\title{
2012 CVET Reviewers
}

Louis Atallah

Ferdinando Auricchio

Evren Azeloglu

Kartik Balachandran

Elias Balaras

Rupak Banerjee

Jared Barber

Ofer Barnea

P. V. Bayly

Eric Berson

Lauren Black

Michael Broomé

Robert Campbell

Krishnan Chandran

Naomi Chesler

Adrian Chester

Ju Chueh

Andre Churchwell

Gabriel Converse

Kevin Costa

Peter Crapo

Josh Cysyk

Eman Daar

Mahsa Dabagh Meshin

Lakshmi Dasi

Tim David

Michael Davis

Curt deGroff

M. Doblare

Gabriele Dubini

Onur Dur

Petter Dyverfeldt

Tino Ebbers

Daniel Einstein

Antonio Ferreira

Richard Figliola

Alberto Figueroa

Ender Finol

John Frangos

Glenn Gaudette

Liang Ge

Don Giddens

Frank Gijsen

Elon Glasberg

Matthew Gounis

Nathan Grenz

Rami Haj-Ali
Josef Halamek

Peter Hammer

Frank Harewood

Einar Heiberg

Leif Rune Hellevik

Luke Herbertson

Stephen Hilbert

Robert Hinton

Hao-Ming Hsiao

Jay Humphrey

Yunlong Huo

Leo Hwa

Jeffrey Jacot

Juan Jimenez

Jorge Jimenez

C. Karmonik

Saeid Kasiri

Yoko Kato

Michael Kelly

Arash Kheradvar

Damir Khismatullin

Hyunggun Kim

Steven Koenig

William Kowalski

Petter Krus

Jan Kucera

Karyn Kunzelman

Catriona Lally

Natalia Landazuri

Trung Le

Zheng Li

John Li

Jun Liao

Baruch Lieber

Ching-Long Lin

Maria Linden

Carol Lucas

Alison Marsden

Timothy Maul

Karen May-Newman

Tim McGloughlin

Prahlad Menon

David Merryman

Francesco Migliavacca

David Molony

Umberto Morbiducci

Liam Morris
Vladimir Muzykantov

Jonathan Mynard

Masanori Nakamura

Jason $\mathrm{Ng}$

Kytai Nguyen

Mette Sofie Olufsen

John Oshinski

George Pantalos

Eric Paterson

Shayn Peirce

Kerem Pekkan

Alan Pelton

Estefanía Peña

Carrie Perlman

João Lemos Pinto

Senol Piskin

Rachael Quinn

Milaca Radisic

Arthur Ritter

Jack Rogers

Arvind Santhanakrishnan

Arthur Sasahara

Chandra Sehgal

Maria Siebes

Dimitrios Sokolis

Philippe Sucosky

John Tarbell

Amy Throckmorton

Lucas Timmins

George Truskey

Akira Tsuda

Kazunori Uemura

Willem van Meurs

Alessandro Veneziani

Sarah Vigmostad

Irene Vignon-Clementel

Alain Vinet

Shigeo Wada

Zhijie Wang

James Warnock

William Weiss

Zhongjun $\mathrm{Wu}$

Ning Yang

Choon Hwai Yap

Zijing Zeng 